\title{
Exhaled volatile organic compounds predict exacerbations of childhood asthma in a 1-year prospective study
}

\author{
Charlotte M. Robroeks ${ }^{1,4}$, Joep J. van Berkel ${ }^{2,4}$, Quirijn Jöbsis ${ }^{1}$, Frederik-Jan van \\ Schooten ${ }^{2}$, Jan W. Dallinga ${ }^{2}$, Emiel F. Wouters ${ }^{3}$ and Edward Dompeling ${ }^{1}$
}

\section{Affiliations:}

'Dept of Paediatric Pulmonology, Maastricht University Medical Centre, Research Institute CAPHRI,

${ }^{2}$ Dept of Health Risk Analysis and Toxicology, Maastricht University, Research Institute NUTRIM, and

${ }^{3}$ Dept of Respiratory Medicine, Maastricht University Medical Centre, Research Institute NUTRIM, Maastricht, The Netherlands.

${ }^{4}$ These authors contributed equally.

\section{Correspondence:}

C.M. Robroeks, Maastricht University Medical Centre, Dept of Paediatric Pulmonology, Research Institute CAPHRI, PO Box 5800, 6202 AZ, Maastricht, The Netherlands.

E-mail: c.bootsma.robroeksagmail.com

ABSTRACT The hypothesis was that prediction of asthma exacerbations in children is possible by profiles of exhaled volatile organic compounds (VOCs), a noninvasive measure of airway inflammation. The aims of the present study were to determine: 1) whether VOCs in exhaled breath are able to predict asthma exacerbations; and 2) the time course and chemical background of the most predictive VOCs.

A prospective study was performed in 40 children with asthma over 1 year. At standard 2-month intervals, exhaled nitric oxide fraction $(F \mathrm{eNO})$, VOC profiles in exhaled breath samples, lung function and symptoms were determined in a standardised way. VOC profiles were analysed by gas chromatographytime-of-flight mass spectrometry.

16 out of 40 children experienced an exacerbation. With support vector machine analysis, the most optimal model of baseline measurements versus exacerbation within patients was based on six VOCs (correct classification 96\%, sensitivity $100 \%$ and specificity 93\%). The model of baseline values of patients with compared to those without an exacerbation consisted of seven VOCs (correct classification 91\%, sensitivity $79 \%$ and specificity 100\%). FeNO and lung function were not predictive for exacerbations.

This study indicates that a combination of different exhaled VOCs is able to predict exacerbations of childhood asthma.

@ERSpublications

Exhaled volatile organic compounds predict exacerbations of childhood asthma in a 1-year prospective study http://ow.ly/kBhGe

Received: Jan 172012 | Accepted after revision: Oct 152012 | First published online: May 032013

Clinical trial: This study is registered at www.clinicaltrials.gov with identifier number NCT00404859.

Support statement: The study was supported by AstraZeneca, MSD, the Jaap Swieringa Foundation and the province of Limburg (the Netherlands).

Conflict of interest: None declared.

Copyright @ERS 2013 


\section{Introduction}

The purpose of asthma treatment is optimal control of the disease [1]. Several cross-sectional surveys have demonstrated that actual levels of asthma control fall far below the goals of national and international guidelines [2-4]. HAMmer et al. [3] reported poor control in 55\% of children with asthma in the Netherlands. Poor asthma control and frequent exacerbations may lead to irreversible, pathological and functional airway changes [5]. Children with asthma often have a poor perception of their complaints relative to normal lung function, which results in under-reporting of symptoms and even underdiagnosis of asthma $[1,3,6]$. Another explanation for poor asthma control may be that although asthma is characterised by chronic airway inflammation, monitoring is not currently performed using measures of airway inflammation $[7,8]$. Indeed, there are indications that exacerbations in asthma may be predicted by sputum eosinophils or by nitric oxide in exhaled breath $[9,10]$.

A relatively new noninvasive technique to assess airway inflammation/oxidative stress in the airways is profiling of volatile organic compounds (VOCs) in exhaled breath. In patients with asthma, production of reactive oxygen species (ROS) is increased [11], which causes lipid peroxidation of cell membranes and subsequent production of VOCs [12]. DALLINGA et al. [13] showed that VOCs in exhaled breath could discriminate between asthmatic children and controls with a high sensitivity and specificity. DRAGONIERI et al. [14] studied "smellprints" of VOCs by means of the "electronic nose" in 10 young adults with mild asthma, 10 older patients with severe asthma and 20 age-matched controls. They found that the electronic nose could discriminate patients with asthma from healthy controls, whereas the distinction between different asthma severities classes was more difficult.

The present longitudinal study investigated the hypothesis that specific VOCs in the exhaled breath of children with asthma are able to predict exacerbations before they are clinically manifest. The specific aims were to investigate: 1) whether VOCs in exhaled breath are able to reliably predict asthma exacerbations and to assess which combination of VOCs is most predictive; and 2) the time course of VOCs during an exacerbation.

\section{Methods \\ Patients}

Children with asthma aged 6-16 years were selected from the outpatient clinic of the Dept of Paediatric Pulmonology, Maastricht University Medical Centre (MUMC+), Maastricht, the Netherlands. All children were known to have had a diagnosis of asthma at our clinic for $\geqslant 6$ months. Asthma was defined based on the following criteria of the Global Initiative for Asthma (GINA) and the Dutch Paediatric Pulmonology Society [1, 15]: 1) recurrent episodes with wheezing, breathlessness, chest tightness or coughing; and 2) one or more occasions with reversible airway obstruction (increase in forced expiratory volume in $1 \mathrm{~s}$ (FEV1) after $400 \mu \mathrm{g}$ salbutamol of $\geqslant 9 \%$ predicted [15]); and/or 3) bronchial hyperresponsiveness to histamine (defined as a provocative concentration of histamine required to induced a $20 \%$ fall in FEV 1 of $\left.<8 \mathrm{mg} \cdot \mathrm{mL}^{-1}\right)$.

All children were treated according to the GINA guideline [1]. Treatment was only adapted on basis of symptoms and lung function. A child was considered atopic when a phadiatop test and/or radioallergosorbent test (RAST) was positive (at least two RAST classes). Exclusion criteria were: 1) diseases that might have interfered with the results of the study (e.g. other chronic inflammatory diseases); 2) inability to properly perform measurements; or 3) active smoking.

\section{Study design}

The study design was a prospective longitudinal study over 1 year. In addition to fixed routine visits to the outpatient clinic every 2 months, patients were asked to visit the outpatient clinic an additional four times during an exacerbation. These extra visits were planned, at most, twice during the study. Extra measurements were planned at day 1, 3 and 5 of the exacerbation, and after return of FEV1 to baseline values and improvement of symptoms (table 1).

\section{Ethics}

This study was approved by the Medical Ethics Committee, MUMC+. The www.clinicaltrials.gov registration number was NCT00404859. All parents gave written informed consent.

\section{Exhaled breath sample collection}

For at least $1 \mathrm{~h}$ before the experiments, no eating or physical exercise were permitted. Subjects were asked to inhale deeply and, subsequently, to exhale into resistance-free Tedlar bags (5 L) as previously described [13, $16,17]$. One to three exhalations were usually sufficient to inflate the bag. Details about exhalation pressure, 
TABLE 1 Study design

Standard

Exacerbation

Standard

\begin{tabular}{llllllllllll}
\hline $\boldsymbol{t}$ & -3 & -2 & -1 & 0 & $\mathrm{e}_{1}$ & $\mathrm{e}_{2}$ & $\mathrm{e}_{3}$ & $\mathrm{e}_{4}$ & +1 & +2 \\
\hline
\end{tabular}

The baseline measurement was defined as the routine measurement before the clinical onset of an asthma exacerbation, denoted by $t=0$. The routine measurements before $t=0$ were coded $t=-1,-2,-3$, etc. The time interval between these measurements was 2 months. During the exacerbation, measurements were denoted by $t=e_{1}, e_{2}, e_{3}$ and $e_{4}$, referring to the additional measurements at day 1,3 and 5 , and at the end of the event, respectively. After the exacerbation, the standard measurements continued (denoted as $t=+1$, +2 , etc.).

sampling time and reproducibility of the procedure are given elsewhere $[16,17]$. The contents of the bag were transferred under standardised conditions onto stainless steel two-bed sorption tubes containing active carbon (Markes International, Llantrisant, UK). VOCs were trapped onto the carbon components until analysis of the sample. In order to analyse the sample, VOCs were released from the tubes by thermal desorption at $270^{\circ} \mathrm{C}$ (UNITY desorption unit; Markes International). VOCs were then separated by gas chromatography (GC) (ThermoFisher Scientific, Austin, TX, USA) and subsequently detected by time-offlight mass spectrometry (TOF-MS) (Thermo Electron Tempus Plus; ThermoFisher Scientific) as previously reported $[13,16,17]$.

\section{Outcome measures}

Primary outcome measure: asthma exacerbation

The occurrence of an asthma exacerbation was the primary outcome measure. The definition of an asthma exacerbation (moderate and severe) was based on the criteria of the American Thoracic Society and European Respiratory Society (ERS): 1) an increase in asthma symptoms (dyspnoea, cough and wheezing) and/or use of short acting $\beta_{2}$-agonists for $\geqslant 2$ days; and/or 2 ) a need for treatment with oral corticosteroids; and/or 3) a need for hospital admission [18].

To recognise an exacerbation at an early stage, AM1 home monitors (CareFusion, Hoechberg, Germany) and modems (HC1; CareFusion) were used. FEV1 measurements, use of rescue medication and the presence and severity of pulmonary symptoms were recorded daily at a fixed time. The intensity of symptoms was scored on a scale from 0 to 3 . All patients were asked to perform the manoeuvres three times within $10 \mathrm{~min}$ and the highest FEV1 (in litres) was stored. Data were sent digitally by telephone modem to a personal computer at the MUMC+ once per week. In the case of deterioration of FEV1 values, and/or an increase in the presence and severity of pulmonary symptoms, patients were called to the hospital for additional measurements and a consultation of the responsible paediatric pulmonologist. At the outpatient clinic, asthma control scores and lung function tests were assessed and breath samples were collected.

Secondary outcome measures

Asthma control score

The asthma control score was assessed 2-monthly by using a validated questionnaire as described previously $[3,4]$. The questionnaire contained questions about chronic airway symptoms, sleep disturbance, limitation of daily activity, asthma attacks, emergency or urgent care visits, and the need for short acting $\beta_{2}$-agonists [3].

\section{Lung function tests}

Short-acting bronchodilators were stopped $\geqslant 8 \mathrm{~h}$ and long-acting bronchodilators $\geqslant 36 \mathrm{~h}$ before testing. Dynamic spirometry was performed by means of the Flowscreen ${ }$ (Carefusion) according to ERS standards [19]. The recorded parameters were: FEV1, forced vital capacity (FVC) and maximum expiratory flow at $50 \%$ of FVC (MEF50), all expressed as a percentage of the predicted normal value [19]. The reversibility to a $\beta_{2}$-agonist was determined $15 \mathrm{~min}$ after inhalation of $400 \mu \mathrm{g}$ salbutamol via a spacer (Volumatic; GlaxoSmithKline, London, UK) at the first, fourth and seventh routine visit.

\section{Analysis of data}

Data acquisition and mining

Analysis of the data output files from GC-TOF-MS was performed in successive steps as previously described in detail [17]. In summary, the first step was to perform peak detection and baseline corrections on all analysis output files. Normalisation of the calculated peak areas was performed using an area scaling factor based on the cumulative area under the detected peaks. Retention times of all samples were assessed. Finally, the output files were merged by combining corresponding compounds based on the degree of 
similarity of the corresponding mass spectra, by determining the match factor values and similarity of retention time. The degree of mass spectrum similarity was calculated using a match factor based on the similarity index as described by STEIN and SCOTT [20].

\section{Analysis}

In order to analyse which VOCs were predictive of an asthma exacerbation, intra- and intersubject comparisons were made. Both comparisons were explored since they might provide different information with regards to the compounds that contribute to an early detection of exacerbations in asthma patients.

\section{Intrasubject comparison}

The first explored comparison was an intrasubject comparison between baseline measurements $(t=0)$ and the first measurement of an exacerbation $\left(t=\mathrm{e}_{1}\right)$. The baseline measurement was defined as the sample taken before the start of the exacerbation (measurement 0 ), on average, 1 month prior to measurement $e_{1}$. The measurements during the exacerbation were chronologically denoted as $e_{1}, e_{2}, e_{3}$ and $e_{4}$. The baseline measurement before $t=0$ was denoted by $t=-1$, the one before that $\mathrm{t}=-2$, etc., as shown in table 1 .

\section{Intersubject comparison}

The second analysis was an intersubject comparison. Baseline samples $(t=0)$ of subjects suffering an exacerbation were compared to baseline measurements of subjects not suffering an exacerbation. Sampling dates of all incorporated samples were chosen as close to one another as possible.

\section{Component selection}

Subsequent component selection and determination of interesting compounds was performed in three ways. 1) Compounds detected in $<8 \%$ of the samples were deleted from the dataset, according to PENN et al. [21]. 2) VOCs with significant different intensities between children with and without exacerbations were selected ( $\mathrm{t}$-tests with Bonferroni correction). Samples at measurement $t=0$ originating from the patients with an exacerbation were compared with samples of the group without an exacerbation (table 1). These samples matched the dates of the $t=0$ samples. 3) Support vector machine (SVM) classification models were used to analyse the ability of VOCs to predict an asthma exacerbation. A SVM is a method in statistics and computer science that analyses data and recognises patterns, and is used for classification and regression analysis. The standard SVM takes a set of input data and predicts, for each given input, which of two possible classes forms the input, making the SVM a nonprobabilistic binary linear classifier. Given a set of training examples, each marked as belonging to one of two categories, an SVM training algorithm builds a model that assigns new examples into one category or the other. An SVM model is a representation of the examples as points in space, mapped so that the examples of the separate categories are divided by a clear gap that is as wide as possible. New examples are then mapped into that same space and predicted to belong to a category based on which side of the gap they fall [22-24].

The SVM approach was chosen for its ability to construct predictive models with large generalisation power even in the case of large dimensionality of the data when the number of observations available for training is low [22]. SVMs are specifically useful since they seek a globally optimised solution and avoid overfitting, allowing for a large number of features or compounds. In order to obtain the best subset of compounds, the attribute selection option implemented in Weka (University of Waikato, Hamilton, New Zealand) was used [23]. Compounds were selected using an SVM attribute evaluator. The attribute evaluator evaluated the worth of a subset of compounds by considering the individual predictive ability of each compound along with the redundancy between them. Preferably, compounds were selected showing high correlations within the class and low intercorrelation. A subset of the highest ranking compounds was implemented into an SVM classifier trained with the sequential minimal optimisation algorithm of PLATT [24]. The SVM classifiers were validated and performance was tested using 10-fold cross-validation in which the entire dataset is split repeatedly into a test set ( $90 \%$ of samples) and a validation set (10\% of samples).

Identification of VOCS

Identification of VOCs was performed by comparing the mass spectra with data from the NIST library (database of the National Institute of Standards and Technology) and by interpretation of the mass spectra by a specialised spectrometrist.

\section{Power analysis}

In order to find clinically meaningful correlations between independent predictors and the exacerbation rate of at least $0.6,35$ children are necessary to assess such a relationship with a two-sided alpha of 0.05 and a power of $98 \%$. During the 12 -month follow-up, a drop-out rate of $10 \%$ was assumed. 40 children with asthma were included in this study. 


\section{Results}

Patient characteristics

Clinical characteristics at inclusion in the study are shown in table 2. The majority of the group was atopic: $29(73 \%)$ out of 40 children. All but one patient was receiving maintenance treatment with inhaled corticosteroids. 38 of the 40 children completed the study, while two patients dropped out. One patient moved out of the region. Before he dropped out, he experienced one exacerbation. The data of this exacerbation were included in the analysis. The other child was not motivated to further participate. She had stable asthma.

\section{Exacerbation rate}

During the 1-year study period, 16 children developed an exacerbation of which 10 were moderate and six were severe. Only three patients developed a second exacerbation. The mean \pm sE time interval between the preceding standard measurement and the onset of an exacerbation was $39 \pm 4$ days.

\section{Volatile organic compounds}

VOCs included in data analysis

In all 39 children with asthma, a total of 3,434 different VOCs were detected in the exhaled breath samples. On average, 343 VOCs were detected per individual. Each subject delivered seven to 15 samples depending on the occurrence of an exacerbation.

\section{Significantly different VOCs}

In total, independent t-tests showed 30 significantly different VOCs after Bonferroni correction $(\mathrm{p}<0.001)$. These VOCs were identified as markers associated with exacerbations.

\section{Timecourse of VOCS}

After selection of the significantly different compounds, these VOCs were tracked in all other samples $(t=-2$ to $e_{4}$ ). Their relative intensity was determined in all samples in order to provide more insight into the timecourse of intensities of these compounds prior to and during exacerbations. As an example, the course of the intensity of three VOCs during an exacerbation are shown in figure 1.

\section{Ability to predict an asthma exacerbation}

All VOCs that were present in $\geqslant 8 \%$ of the samples were included in the SVM analyses in order to assess the ability of VOCs to predict an asthma exacerbation [16, 17]. Results of SVM models are based on VOCs considered together. The relationship between the number of different VOCs in the SVM model and the

\section{TABLE 2 Clinical characteristics of the asthmatic children at inclusion of the study}

$\begin{array}{lc}\text { Age years } & 10.7 \pm 0.4 \\ \text { Weight kg } & 38.8 \pm 2.0 \\ \text { Height } \mathbf{~ c m} & 142.3 \pm 2.4 \\ \text { Males/females } & 29 / 11 \\ \text { ACQ score Irange) } & 27.6 \pm 2.3(9-64) \\ \text { Lung function indices } & \\ \text { Reversibility, increase in FEV1 \% pred } & 5.9 \pm 1.1 \\ \text { FEV1\% pred } & 99.6 \pm 2.2 \\ \text { FEV1/VC \% } & 83.9 \pm 1.5 \\ \text { FVC \% pred } & 99.2 \pm 2.4 \\ \text { MEF50 \% pred } & 82.6 \pm 3.8 \\ \text { Atopy yes/no } & 29 / 11 \\ \text { Total IgE kU. L }{ }^{-1} & 493.3 \pm 120.7 \\ \text { Active eczema } & 7(18) \\ \text { Allergic rhinitis } & 4(10) \\ \text { Treatment } & \\ \text { Dose of inhaled budesonide or equivalent } \mu \mathrm{gg} & 587 \pm 53 \\ \text { Long-acting } \beta_{2} \text {-agonist } & 21(53) \\ \text { Antihistamines } & 9(23) \\ \text { Leukotriene receptor antagonist } & 8(20)\end{array}$

Data are presented as mean $\pm \mathrm{SEM}, \mathrm{n}$ or $\mathrm{n}(\%)$, unless otherwise stated. $\mathrm{ACQ}$ : Asthma Control Questionnaire; FEV1: forced expiratory volume in $1 \mathrm{~s}$; \% pred: \% predicted; VC: vital capacity; FVC: forced vital capacity; MEF50: mean expiratory flow at 50\% of FVC; Ig: immunoglobulin. 

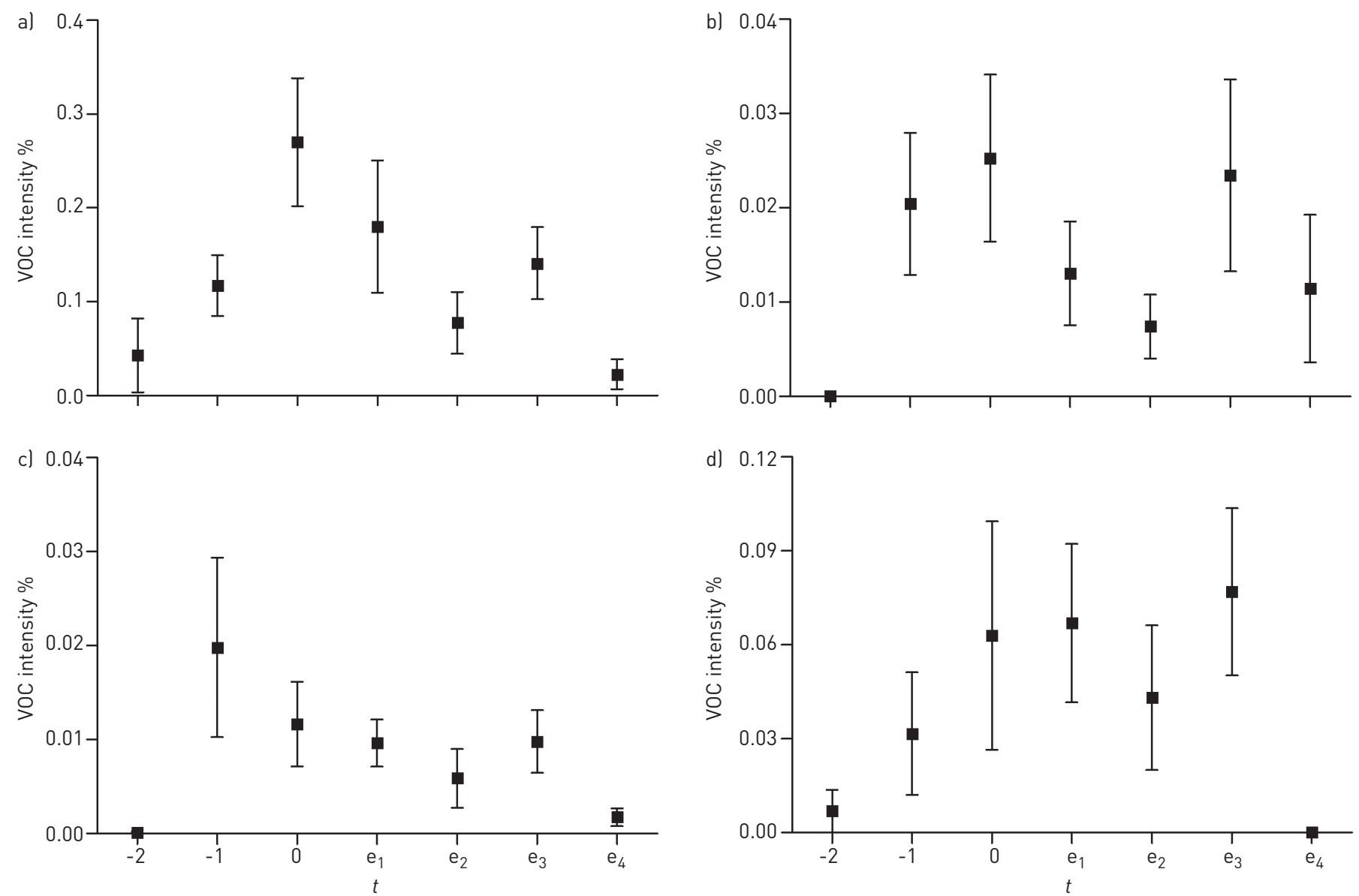

FIGURE 1 Intensity of four significantly different volatile organic compounds (VOCs) demonstrating their time course before and during an asthma exacerbation. a) $p$-xylene (Friedman test $\mathrm{p}=0.005$ ); b) 8,11-octadecadiynoic acid methylester (Friedman test $\mathrm{p}=0.01$ ); $\mathrm{c}$ ) 3,6-octadecadiynoic acid methylester (Friedman test $\mathrm{p}=0.004)$; ) octadecane (Friedman test $\mathrm{p}=0.01$ ). Data are presented as mean \pm SE. For definitions of the time points $(t)$, see table 1 .

corresponding sensitivity and specificity is shown in tables 3 and 4. In the intrasubject comparison (baseline versus exacerbation within patients), the optimally performing SVM was based on six VOCs. Exacerbations were correctly classified in $96 \%$ of samples (sensitivity $100 \%$, specificity $93 \%$, positive predicted value $89 \%$ and negative predicted value $100 \%$ ) as shown in table 3 . The optimally performing SVM of intersubject comparison (baseline resulting in exacerbation versus baseline not resulting in exacerbation, between patients) was based on seven VOCs. A correct classification rate of $91 \%$ was found with a corresponding sensitivity and specificity of $78 \%$ and $100 \%$ (positive predicted value $100 \%$ and negative predicted value

TABLE 3 The relationship between the number of volatile organic compounds (VOCs) in the support vector machine model and the prediction of exacerbations: intrasubject comparison

\begin{tabular}{lccc} 
VoCs n & Sensitivity $\%$ & Specificity $\%$ & Correct classification $\%$ \\
\hline $\mathbf{9}$ & 100 & 100 & 100 \\
$\mathbf{8}$ & 100 & 100 & 100 \\
$\mathbf{7}$ & 100 & 93 & 96 \\
$\mathbf{6}$ & 100 & 93 & 96 \\
$\mathbf{5}$ & 100 & 79 & 89 \\
$\mathbf{4}$ & 100 & 71 & 82 \\
$\mathbf{3}$ & 100 & 64 & 75 \\
$\mathbf{2}$ & 100 & 50 & 64 \\
$\mathbf{1}$ & 100 & 29 & 66
\end{tabular}

An optimal model contained six VOCs and had a sensitivity of $100 \%$, a specificity of $93 \%$, a positive predicted value of $89 \%$ and a negative predicted value of $100 \%$ (bold). 
TABLE 4 The relationship between the number of volatile organic compounds (VOCs) in the support vector machine model and the prediction of exacerbations: intersubject comparison

$\begin{array}{llll}\text { VOCs } n & \text { Sensitivity } \% & \text { Specificity } \% & \text { Correct classification } \%\end{array}$

\begin{tabular}{llcl}
\hline $\mathbf{9}$ & 79 & 95 & 88 \\
$\mathbf{8}$ & 71 & 95 & 85 \\
$\mathbf{7}$ & $\mathbf{7 9}$ & $\mathbf{1 0 0}$ & $\mathbf{9 1}$ \\
$\mathbf{6}$ & 100 & 85 \\
$\mathbf{5}$ & 64 & 100 & 88 \\
$\mathbf{4}$ & 71 & 100 & 85 \\
$\mathbf{3}$ & 64 & 100 & 76 \\
$\mathbf{2}$ & 43 & 100 & 73 \\
$\mathbf{1}$ & 36 & 100 & 67
\end{tabular}

An optimal model contained seven VOCs and had a sensitivity of $79 \%$, a specificity of $100 \%$, a positive predicted value of $100 \%$ and a negative predicted value of $86 \%$ (bold).

$86 \%$ ), respectively (table 4). Neither FeNO nor lung function were no significant predictors of exacerbations. Univariate Cox regression analysis of the time until an exacerbation showed that neither FeNO nor FEV1 were significant predictors of an asthma exacerbation $(\mathrm{p}=0.43$ and $\mathrm{p}=0.60$, respectively).

Identification of VOCs

It was possible to identify five out of six VOCs of the optimally discriminating SVM model for intrasubject comparison and six out of seven VOCs for intersubject comparison (tables 5 and 6).

\section{Discussion}

This study indicates that VOCs in exhaled breath are able to predict asthma exacerbations in children. We found that a combination of six or seven VOCs was able to predict exacerbations both between and within patients with a high sensitivity and specificity. It was also possible to identify VOCs that significantly "tracked" the course of an exacerbation. The identified VOCs were mainly classified as hydrocarbons. Both FeNO and lung function had no significant predictive value for exacerbations in this study.

To our knowledge, this is the first longitudinal study to analyse the ability of VOCs in exhaled breath to predict exacerbations of childhood asthma, and to study the course of VOCs during an exacerbation. In a recent study, IBRAHIM et al. [25] studied the potential of VOC profiling to assess asthma diagnosis, sputum inflammatory cell profile and asthma control in 35 adult asthmatics and 23 matched controls. They demonstrated that VOC profiles were able to accurately identify patients with sputum eosinophilia and poor disease control with cross-validated accuracies of $83 \%$ and $80 \%$, respectively. In a cross-sectional study of 120 children (57 controls and 63 asthma patients), we found a good discrimination between asthma and controls based on an optimal combination of eight VOCs in exhaled breath [13]. In adults, DRAGONIERI et al. [14] assessed VOC patterns in exhaled breath by means of the electronic nose. They found that asthma could be differentiated from controls but the discrimination between different degrees of asthma severity was not satisfactory. In contrast, to GC-TOF-MS used in our study, the electronic nose in the study of DRAGONIERI et al. [14] was used to assess smellprints of different VOCs. These techniques are fundamentally different, since the smellprints are based on pattern recognition instead of assessing single mass spectra. The disadvantage of smellprints is that it may not measure specific VOCs that are important for the discrimination. The advantage of the approach used in the present study is that the most predictive VOCs are detected and that the chemical background of the compounds can be elucidated. This information can be used in the future to construct an electronic nose that is specifically useful for a certain disease (e.g. childhood asthma) and a specific clinical question (e.g. the early detection of exacerbations).

Regular 2-month assessments of FeNO in this study were not predictive of exacerbations.

In a 6-month study by FRITSCH et al. [26] of 47 children with mild-to-moderate asthma, FeNO at a cut-off point of $29 \mathrm{ppb}$ was most predictive of an exacerbation with a sensitivity of $80 \%$ and a specificity of $60 \%$. In the inhaled corticosteroid withdrawal study of PijNENBURG et al. [27], FeNO values $>49$ ppb 4 weeks after discontinuation had a sensitivity for an asthma relapse of $71 \%$ and a specificity of $93 \%$. In nonatopic patients with asthma, FeNO may not be elevated [28]. The advantage of VOC analysis in exhaled breath is that several inflammatory markers are measured in exhaled breath simultaneously and that the most predictive biomarkers can be selected, as indicated by the high sensitivity and specificity of the combination of VOCs in our study. 
TABLE 5 Identified compounds as implemented into the most optimal support vector machine classifiers of intrasubject comparison

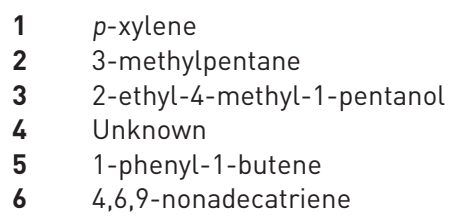

It is relevant to discuss several elements of our study. First, a group of 40 children with asthma was included, of which 16 children experienced an exacerbation during the year of follow-up. Although internal validation was performed in our study by 10 -fold cross-validation and exacerbations could be predicted with good sensitivity and specificity, external validation of our study in larger study populations will be necessary to confirm the optimal combination of VOCs [29]. 29 of the 40 children were atopic but our number of children was too low to perform a post hoc analysis in this group.

Secondly, the presence of VOCs may be influenced by intrinsic (sex, age, weight, atopy and lung function) and extrinsic factors (e.g. ambient air or medication). Most of these intrinsic factors were well controlled in our study. Moreover, no relationship was found between these intrinsic factors, medication and exacerbations during the study. This almost rules out a possible influence of these factors on the results of this study. Currently, there is no consensus on the role of VOCs in ambient air on VOC profiling in exhaled breath. The children were all measured in the same room for each session and, therefore, were all influenced by ambient air in a comparable way. As reported previously by VAN BERKEL et al. [17] and DALLINGA et al. [13], we did not correct our measurements for chemical background appearing in the samples. This is because of the fact that it would not be possible to correct for the complex interdependencies between excretion and uptake of VOCs by easily subtracting the inhaled from the exhaled air. Moreover, background noise will be randomly distributed between subjects' samples and will, thus, neither exert any discriminatory power nor interfere with the outcome of the analyses. We are aiming with discriminative analysis to select only those compounds that are specific for the disease or condition and should, thus, principally not depend on background chemicals. An earlier report by our research group concluded that the procedure followed for sampling, chemical analysis, data handling and accurate data mining was highly reproducible [17]. In addition, chemical structures and metabolites of medication used were not reflected in the significantly predictive VOCs.

Thirdly, the origin of the identified VOCs remains in some instances uncertain. We were able to identify the majority of the predicting VOCs.

We hypothesise that discriminating VOCs reflect the degree of airway inflammation and oxidative stress. Saturated hydrocarbons (VOCs) in exhaled breath are formed during lipid peroxidation of fatty acid components of cell membranes. This process is triggered by ROS [17, 30-35].

In the future, it is relevant to study whether titration of anti-inflammatory treatment on the basis of VOCs is helpful in the prevention of exacerbations of asthma. In addition, studies are necessary to clarify the biochemical origin, pathophysiological function and optimal way of measuring predictive VOCs.

Breath analysis mirrors biochemical and immunological processes in the human lung. The present study indicates that VOC profiling is able to predict asthma exacerbations in children. Future studies are needed for external validation of the results in larger patient samples, to explore the nature of these VOCs and to investigate whether better asthma control can be achieved when exhaled breath analysis is included as a parameter in daily clinical practice.

TABLE 6 Identified compounds as implemented into the most optimal support vector machine classifiers of intersubject comparison

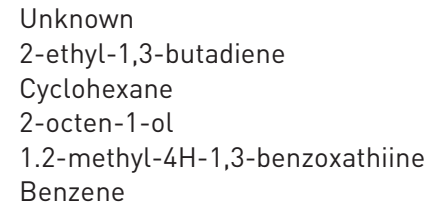




\section{References}

1 Global Initiative for Asthma. Pocket guide for asthma management and prevention in children 5 years and younger. www.ginasthma.org/local/uploads/files/GINA_Under5_Pocket_20091_1.pdf Date last accessed: May 13, 2013. Date last updated: May 2009.

2 Gustafsson PM, Watson L, Davis KJ, et al. Poor asthma control in children: evidence from epidemiological surveys and implications for clinical practice. Int J Clin Pract 2006; 60: 321-334.

3 Hammer SC, Robroeks CM, van Rij C, et al. Actual asthma control in a paediatric outpatient clinic population: do patients perceive their actual level of control? Pediatr Allergy Immunol 2008; 19: 626-633.

4 Rabe KF, Adachi M, Lai CK, et al. Worldwide severity and control of asthma in children and adults: the global asthma insights and reality surveys. J Allergy Clin Immunol 2004; 114: 40-47.

5 O'Connell EJ. Optimizing inhaled corticosteroid therapy in children with chronic asthma. Pediatr Pulmonol 2005; 39: 74-83.

6 van Gent R, van Essen-Zandvliet LE, Rovers MM, et al. Poor perception of dyspnoea in children with undiagnosed asthma. Eur Respir J 2007; 30: 887-891.

$7 \quad$ Kharitonov SA, Barnes PJ. Biomarkers of some pulmonary diseases in exhaled breath. Biomarkers 2002; 7: 1-32.

8 Kips JC, Kharitonov SA, Barnes PJ. Noninvasive assessment of airway inflammation in asthma. Eur Respir Monogr 2003; 13: 164-179.

9 Zacharasiewicz A, Wilson N, Lex C, et al. Clinical use of noninvasive measurements of airway inflammation in steroid reduction in children. Am J Respir Crit Care Med 2005; 171: 1077-1082.

10 Pijnenburg MW, de Jongste JC. Exhaled nitric oxide in childhood asthma: a review. Clin Exp Allergy 2008; 38: 246-249.

11 Rahman I, Kelly F. Biomarkers in breath condensate: a promising new non-invasive technique in free radical research. Free Radic Res 2003; 37: 1253-1266.

12 Kinnula VL. Production and degradation of oxygen metabolites during inflammatory states in the human lung. Curr Drug Targets Inflamm Allergy 2005; 4: 465-470.

13 Dallinga JW, Robroeks CMHHT, Van Berkel JJ, et al. Analysis of volatile organic compounds in exhaled breath as a diagnostic tool for asthma in children. Clin Exp Allergy 2010; 40: 68-76.

14 Dragonieri S, Schot R, Mertens BJ, et al. An electronic nose in the discrimination of patients with asthma and controls. J Allergy Clin Immunol 2007; 120: 856-862.

15 De Jongste JC, Vrijlandt EJLE. Astma monitoring bij kinderen. [Asthma monitoring in children.] http:// professionals.gsk.nl/content/dam/Health/nl_NL/HCP_Home/therapiegebieden/Respiratoir_en_Allergie/Abstract\%20-\% 20Astma\%20monitoring\%20E-health.pdf Date last accessed: May 13, 2013. Date last updated: 2008.

16 Robroeks CM, van Berkel JJ, Dallinga JW, et al. Metabolomics of volatile organic compounds in cystic fibrosis patients and controls. Pediatr Res 2010; 68: 75-80.

17 Van Berkel JJ, Dallinga JW, Moller GM, et al. Development of accurate classification method based on the analysis of volatile organic compounds from human exhaled air. J Chromatogr B Analyt Technol Biomed Life Sci 2008; 861: 101-107.

18 Reddel HK, Taylor DR, Bateman ED, et al. An Official American Thoracic Society/European Respiratory Society statement: asthma control and exacerbations standardizing endpoints for clinical asthma trials and clinical practice. Am J Respir Crit Care Med 2009; 180: 59-99.

19 Quanjer PH, Tammeling GJ, Cotes JE, et al. Symbols, abbreviations and units. Working Part Standardization of Lung Function Tests, European Community for Steel and Coal. Eur Respir J 1993; 6: Suppl. 16, S85-S100.

20 Stein SE, Scott DR. Optimization and Testing of Mass-Spectral Library Search Algorithms for Compound Identification. J Am Soc Mass Spectr 1994; 5: 859-866.

21 Penn DJ, Oberzaucher E, Grammer K, et al. Individual and gender fingerprints in human body odour. $J$ R Soc Interface 2007; 4: 331-340.

22 Guyon I, Weston J, Barnhill S, et al. Gene selection for cancer classification using support vector machines. Machine Learning 2002; 46: 389-422.

23 Frank E, Hall M, Trigg L, et al. Data mining in bioinformatics using Weka. Bioinformatics 2004; 20: 2479-2481.

24 Platt JC. Fast training of support vector machines using sequential minimal optimization. Cambridge, MIT Press, 1998.

25 Ibrahim B, Basanta M, Cadden $\mathrm{P}$, et al. Non-invasive phenotyping using exhaled volatile organic compounds in asthma. Thorax 2011; 66: 804-809.

26 Fritsch M, Uxa S, Horak F Jr, et al. Exhaled nitric oxide in the management of childhood asthma: a prospective 6months study. Pediatr Pulmonol 2006; 41: 855-862.

27 Pijnenburg MW, Hofhuis W, Hop WC, et al. Exhaled nitric oxide predicts asthma relapse in children with clinical asthma remission. Thorax 2005; 60: 215-218.

28 Silvestri M, Sabatini F, Spallarossa D, et al. Exhaled nitric oxide levels in non-allergic and allergic mono- or polysensitised children with asthma. Thorax 2001; 56: 857-862

29 Steyerberg EW, Bleeker SE, Moll HA, et al. Internal and external validation of predictive models: a simulation study of bias and precision in small samples. J Clin Epidemiol 2003; 56: 441-447.

30 Sanchez JM, Sacks RD. Development of a multibed sorption trap, comprehensive two-dimensional gas chromatography, and time-of-flight mass spectrometry system for the analysis of volatile organic compounds in human breath. Anal Chem 2006; 78: 3046-3054.

31 Ashley DL, Bonin MA, Hamar B, et al. Using the blood concentration of 2,5-dimethylfuran as a marker for smoking. Int Arch Occup Environ Health 1996; 68: 183-187.

32 Corradi M, Pignatti P, Manini P, et al. Comparison between exhaled and sputum oxidative stress biomarkers in chronic airway inflammation. Eur Respir J 2004; 24: 1011-1017.

33 Lex C, Zacharasiewicz A, Payne DN, et al. Exhaled breath condensate cysteinyl leukotrienes and airway remodeling in childhood asthma: a pilot study. Respir Res 2006; 7: 63.

34 Paredi P, Kharitonov SA, Leak D, et al. Exhaled ethane, a marker of lipid peroxidation, is elevated in chronic obstructive pulmonary disease. Am J Respir Crit Care Med 2000; 162: 369-373.

35 Phillips M, Herrera J, Krishnan S, et al. Variation in volatile organic compounds in the breath of normal humans. JChromatogr B Biomed Sci Appl 1999; 729: 75-88. 